\title{
Scientometrics rút bài báo sử dụng dữ liệu từ danh sách Beall
}

\author{
- sciencevietnam $\leftleftarrows$ (1) September 08,2021
}

ENHANCED BY Google

SSHPA (08-09-2021) — Mới đây, tạp chí Scientometrics đã quyết định rút bài báo có tựa đề "Predatory publishing in Scopus: evidence on cross-country differences" [1,2]. Theo thông báo rút bài [2], tổng biên tập Scientometrics đưa ra quyết định này vì các kết quả trong bài không đảm bảo. Quá trình bình duyệt sau xuất bản cũng đánh giá phân tích hồi quy không đầy đủ. Đáng chú ý, cả hai tác giả của bài đều không đồng ý với quyết định này.

Bài báo Predatory publishing in Scopus: evidence on cross-country differences tìm kiếm các tạp chí có tên trong cả danh sách Beall về các tạp chí săn mồi và cơ sở dữ liệu Scopus. Các tác giả cho thấy có 324 tạp chí và hơn 165 ngàn bài báo trong giai đoạn 2015-2017 thuộc cả 2 danh mục [1]. Kết quả này sau đó cũng được tạp chí Nature đưa tin [3].

Retraction Note | Published: 06 September 2021

\section{Retraction Note to: Predatory publishing in Scopus: evidence on cross-country differences}

\author{
Vít Macháček $\square$ \& $\underline{\text { Martin Srholec }}$ \\ Scientometrics (2021) $\mid$ Cite this article \\ 259 Accesses 33 Altmetric $\mid \underline{\text { Metrics }}$ \\ (3) The Original Article was published on 07 February 2021
}

\section{Retraction Note to: Scientometrics (2021) 126:1897-1921 https://doi.org/10.1007/s11192-020-03852-4}

Về vụ việc rút bài của nghiên cứu này, trang RetractionWatch cho biết Frontiersnhà xuất bản có tạp chí được nhắc tới trong nghiên cứu—đã đề nghị Scientometrics rút bài vì các vấn đề xung quanh danh sách Beall [4]. Mặc dù là một trong những danh mục đầu tiên về tạp chí săn mồi, danh sách Beall đã phải dừng hoạt động vì các vấn đề về phương pháp luận, dữ liệu và sự minh bạch.

Phản hồi lại lời đề nghị này, các tác giả cho biết danh sách Beall hiện vẫn được sử dụng rộng rãi để xác định các tạp chí có khả năng là tạp chí săn mồi. Chính vì vậy, không có lý do gì để rút bài. 
Sau đó, tạp chí Scientometrics đã gửi bài báo đi phản biện sau xuất bản. Cuối cùng, quyết định rút bài đã được đưa ra. Mặc dù vậy, quyết định này cũng gây ra bức xúc cho hai tác giả của bài báo và cả các thành viên trong ban biên tập. Một thành viên ban biên tập Scientometrics cho biết ông săn sàng từ chức để phản đối quyết định này [4].

Có thể thấy, vấn đề rút bài, và quy trình rút bài hiện nay vẫn còn nhiều vấn đề và cần được cải tổ mạnh mẽ [5,6].

\section{Tài liệu tham khảo:}

[1] Macháček V, Srholec M. (2021). RETRACTED ARTICLE: Predatory publishing in Scopus: evidence on cross-country differences. Scientometrics, 126, 1897-1921, DOI: $10.1007 / \mathrm{s} 11192-020-03852-4$

[2] Macháček V, Srholec M. (2021). Retraction Note to: Predatory publishing in Scopus: evidence on cross-country differences. Scientometrics, DOI:

10.1007/s11192-021-04149-w

[3] Chawla DS. (2021). Hundreds of 'predatory' journals indexed on leading scholarly database. Nature, DOI: 10.1038/d41586-021-00239-0

[4] Oransky I. (2021). Authors object after Springer Nature journal cedes to publisher Frontiers' demand for retraction. RetractionWatch. URL: https://retractionwatch.com /2021/09/07/authors-object-after-springer-nature-journal-cedes-to-publisherfrontiers-demand-for-retraction/

[5] Vuong QH. (2020). Reform retractions to make them more transparent. Nature, 582(7811), 149.

[6] da Silva JAT, Vuong QH. (2021). Fortification of retraction notices to improve their transparency and usefulness. Learned Publishing, 34(AOP), DOI: 10.1002/leap.1409

(1) Last modified: 9/8/2021 4:03 PM

Views: 2
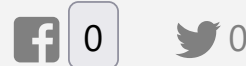

\section{Bài liên quan:}

- Bài nghiên cứu phải xin rút bài tới hai lần

- Tác giả Việt nêu quan điểm về việc rút bài báo khoa học trên Nature

- Lỗi tại Excel

- "Gót chân Achilles" của xuất bản khoa học

- Bình duyệt học thuật cho các tạp chí "săn mồi"

\section{Ý kiến bạn đọc (0):}

Comment 
(C) 2018 - 2021 EASE Vietnam SciComm System. All rights reserved. Powered by Vuong \& Associates. Built to serve the social sciences and humanities. 